DOI: https://doi.org/10.14311/TPFM.2022.026

\title{
ON NUMERICAL SIMULATION OF FLUID-STRUCTURE INTERACTION PROBLEMS BY DISCONTINUOUS GALERKIN AND FINITE VOLUME TECHNIQUES
}

\author{
O. Winter ${ }^{1,2}$, P. Sváček ${ }^{1,2}$
}

\author{
${ }^{1}$ Department of Technical Mathematics, Faculty of Mechanical Engineering, Czech Technical \\ University in Prague, Karlovo náměstí 13, 121 35, Prague 2, Czech Republic \\ ${ }^{2}$ Center of Aviation and Space Research, Faculty of Mechanical Engineering, University of \\ Czech Technical University in Prague, Technická 4, 16607 Prague 6, Czech Republic
}

\begin{abstract}
This work focuses on application of a high-order accurate scheme for coupled fluid-structure interaction problems. A simplified mathematical model is considered consisting of the NavierStokes equations describing the compressible fluid flow and the equation of motion describing the movement of a structure. The fluid flow is discretized using a discontinuous Galerkin method on unstructured triangular meshes, and the structure uses high-order explicit RungeKutta method. Results obtained by presented scheme and OpenFOAM's implementation of a finite volume scheme are compared.
\end{abstract}

Keywords: discontinuous Galerkin method, finite volume method, fluid-structure interaction.

\section{Introduction}

Many important scientific and engineering problems require analysis of a fluid-structure interaction (FSI) phenomena. For example, an aeroelastic flutter can produce large and potentially destructive vibrations in aircraft [11], turbines [1], and other structures [6] or biological applications such as study of a fluid flow inside human vocal tract [8].

The aim of this study is to compare a high order discontinuous Galerkin (DG) and a second order finite volume (FV) schemes applied to the fluid-structure interaction problems. The main purpose of this comparison is to investigate the grid requirements and the sufficient number of degrees of freedom (DoF) to obtain adequate approximation. Two benchmark cases for the verification of DG scheme and the comparison of DG and FV schemes are considered: (I) the flow around the cylinder with one degree of freedom which allows to the cylinder vibrate in the vertical direction according to the flow field, and (II) the flow around flexibly supported airfoil with two degrees of freedom coupled to the fluid flow.

\section{Mathematical Model}

The mathematical model consists of the Navier-Stokes equations governing the motion of a compressible fluid flow coupled to a rigid body dynamics, i.e., a movement of a structure, described by a system of an ordinary differential equations. Arbitrary Lagrangian Eulerian (ALE) method is used to treat the deformable domain, see e.g. [11].

\subsection{Fluid Flow Model}

Let us denote by $\Omega_{t} \subset \mathbb{R}^{2}$ the bounded computational domain occupied by the compressible fluid at any time $t \in(0, T), T>0$, see Figure 1 . The motion of the viscous compressible fluid in computational domain $\Omega_{t}$ is described by the Navier-Stokes equations, see e.g. [2], written in the 


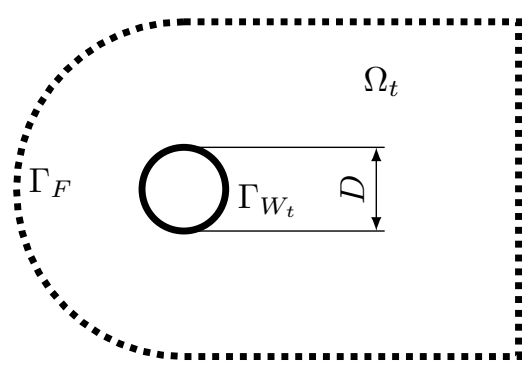

(a) Flow around the Cylinder

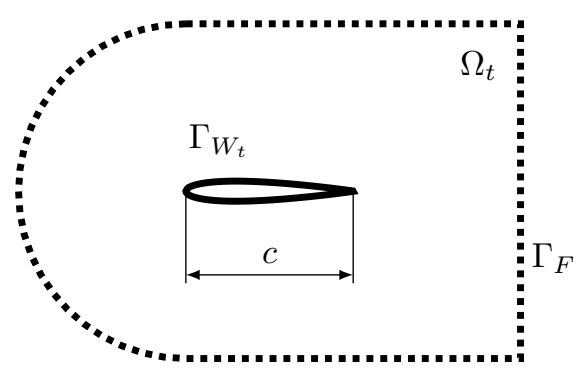

(b) Flow around NACA 0012 airfoil

Figure 1: Sketches of the computational domains $\Omega_{t}$

conservative form as

$$
\begin{gathered}
\frac{\partial}{\partial t}(\varrho)+\sum_{i=1}^{2} \frac{\partial}{\partial x_{i}}\left(\varrho v_{i}\right)=0 \\
\frac{\partial}{\partial t}\left(\varrho v_{i}\right)+\sum_{j=1}^{2} \frac{\partial}{\partial x_{j}}\left(\varrho v_{i} v_{j}+p \delta_{i j}\right)=\sum_{j=1}^{2} \frac{\partial}{\partial x_{j}}\left(\tau_{i j}\right), \quad \text { for } i=1,2, \\
\frac{\partial}{\partial t}(\varrho E)+\sum_{j=1}^{2} \frac{\partial}{\partial x_{j}}\left(\varrho v_{j} E+v_{j} p\right)=\sum_{j=1}^{2} \frac{\partial}{\partial x_{j}}\left(-q_{j}+v_{i} \tau_{i j}\right)
\end{gathered}
$$

where $\varrho$ is the fluid density, $p$ is the pressure, $v_{1}, v_{2}$ are the velocity components of the velocity vector $\boldsymbol{v}$, and $E$ is the total energy. The components of the viscous stress tensor $\boldsymbol{\tau}$ and the heat flux $\boldsymbol{q}$ are given by

$$
\tau_{i j}=\mu\left(\frac{\partial v_{i}}{\partial x_{j}}+\frac{\partial v_{j}}{\partial x_{i}}-\sum_{k=1}^{2} \frac{2}{3} \frac{\partial v_{k}}{\partial x_{k}} \delta_{i j}\right)
$$

and

$$
q_{i}=\frac{\mu}{\operatorname{Pr}} \frac{\partial}{\partial x_{i}}\left(E+\frac{p}{\varrho}-\sum_{j=1}^{2} \frac{1}{2} v_{j} v_{j}\right),
$$

where $\mu$ is the dynamic viscosity and Pr is the Prandtl number (assumed to be constant $\operatorname{Pr}=0.72$ ). For an ideal gas the pressure $p$ is given by

$$
p=(\gamma-1) \varrho\left(E-\frac{1}{2} \sum_{i=1}^{2} v_{i} v_{i}\right),
$$

where $\gamma$ is the adiabatic gas constant ( $\gamma$ is set to 1.4). Imposed boundary conditions are either subsonic free-stream at the far field, or adiabatic no-slip conditions at the boundaries of the structure, i.e., $\left.\boldsymbol{v}\right|_{\Gamma_{W_{t}}}$ is equal to the velocity of the structure, see Figure 1. System (1) is supplemented with suitable initial conditions.

\subsection{Structure Model}

Two different models for each test case are considered. 


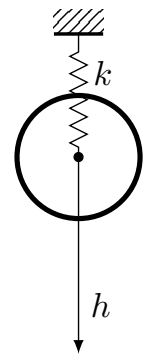

(a) Flow around Cylinder

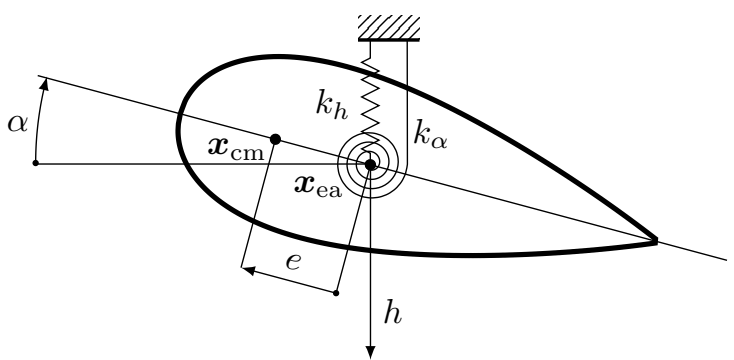

(b) Flow around NACA 0012 airfoil

Figure 2: Sketches of structure models

For the first case, see Figures $1 \mathrm{a}$ and $2 \mathrm{a}$, the model consists of cylinder vibrating in vertical direction according to

$$
m \ddot{h}+d \dot{h}+k h=-L,
$$

where $m$ is the oscillating mass of the system, $d$ and $k$ denote the mechanical damping and stiffness of the oscillator unit, respectively, $h$ is the displacement of the oscillator (downwards positive) and $L$ is the force exerted by the fluid on the structure in the transverse direction, i.e., aeroelastic lift force (upwards positive).

And for the second case, see Figures $1 \mathrm{~b}$ and 2b, the model consists of NACA 0012 airfoil vibrating in vertical direction and rotating around an elastic axis $\boldsymbol{x}_{e a}$ according to the system of non-linear ordinary differential equation, i.e.

$$
\begin{aligned}
m \ddot{h}+k_{h} h+S_{\alpha} \ddot{\alpha} \cos \alpha-S_{\alpha} \dot{\alpha}^{2} \sin \alpha & =-L, \\
S_{\alpha} \ddot{h} \cos \alpha+I_{\alpha} \ddot{\alpha}+k_{\alpha} \alpha & =M,
\end{aligned}
$$

where $\alpha$ is the rotational displacement around the elastic axis (clockwise positive) and $h$ is vertical displacement of the elastic axis (downwards positive), see e.g. [5]. In (6), $m$ is the mass of the airfoil, $S_{\alpha}$ is the static moment around the elastic axis, $I_{\alpha}$ is the inertia moment around the elastic axis, $k_{h}$ is the bending stiffness, and $k_{\alpha}$ is the torsional stiffness. $L$ and $M$ denotes the aerodynamic lift force (upwards positive) and the aerodynamic torsional moment (clockwise positive), respectively.

Fluid flow model (1) is coupled with the rigid body model in both cases via $L$ and in the second case also via $M$ in the following manner, i.e.

$$
\begin{aligned}
& L=-l \int_{\Gamma_{W_{t}}} \sum_{j=1}^{2}\left(\tau_{2 j}-p \delta_{2 j}\right) n_{j} d S, \\
& M=l \int_{\Gamma_{W_{t}}} \sum_{i, j=1}^{2}\left(\tau_{i j}-p \delta_{i j}\right) n_{j} r_{i}^{\text {ort }} d S,
\end{aligned}
$$

where

$$
r_{1}^{\mathrm{ort}}=-\left(x_{2}-x_{2}^{\mathrm{ea}}\right), \quad r_{2}^{\mathrm{ort}}=x_{1}-x_{1}^{\mathrm{ea}},
$$

and $l$ is the depth of the rigid body section and $\boldsymbol{n}=\left(n_{1}, n_{2}\right)$ is the unit outer normal to $\partial \Omega_{t}$ on $\Gamma_{W_{t}}$ (pointing into the airfoil).

\section{Numerical Approximation}

\subsection{Finite Volume Method}

Finite volume scheme used for this study is based on OpenFOAM's implementation, see [10]. The system of equations (1) is solved via matrix-free lower-upper symmetric Gauss-Seidel (LUSGS) method, see [3]. The convective terms in equations are discretized using Roe numerical flux together with a piece-wise linear reconstructions and the diffusive terms via limited linear scheme. The system is evolved in time with aid of the second order backward differentiation formula, for more details see e.g. [11] or [3]. 


\subsection{Discontinuous Galerkin Method}

For the purpose of the discretization via discontinuous Galerkin method the system (1) is rewritten into the system of first order equations, i.e.,

$$
\begin{aligned}
\frac{\partial}{\partial t}(\boldsymbol{u})+\nabla \cdot \boldsymbol{F}^{i}(\boldsymbol{u})-\nabla \cdot \boldsymbol{F}^{v}(\boldsymbol{u}, \boldsymbol{g}) & =\mathbf{0} \\
\nabla \boldsymbol{u} & =\boldsymbol{g},
\end{aligned}
$$

where $\boldsymbol{g}$ is auxiliary gradient variable, $\boldsymbol{u}=\left[\varrho, \varrho v_{1}, \varrho v_{2}, \varrho E\right]^{T}$ is the solution vector, $\boldsymbol{F}^{i}$ is inviscid flux given as

$$
\boldsymbol{F}^{i}(\boldsymbol{u})=\left[\begin{array}{cc}
\varrho v_{1} & \varrho v_{2} \\
\varrho v_{1} v_{1} & \varrho v_{2} v_{1} \\
\varrho v_{1} v_{2} & \varrho v_{2} v_{2} \\
(\varrho E+p) v_{1} & (\varrho E+p) v_{2}
\end{array}\right]
$$

and $\boldsymbol{F}^{v}$ is viscous flux given as

$$
\boldsymbol{F}^{v}(\boldsymbol{u}, \boldsymbol{g})=\left[\begin{array}{cc}
0 & 0 \\
\tau_{11}(\boldsymbol{g}) & +\tau_{12}(\boldsymbol{g}) \\
\tau_{21}(\boldsymbol{g}) & +\tau_{22}(\boldsymbol{g}) \\
-q_{1}(\boldsymbol{u})+v_{1} \tau_{11}(\boldsymbol{g})+v_{2} \tau_{21}(\boldsymbol{g}) & -q_{2}(\boldsymbol{u})+v_{1} \tau_{12}(\boldsymbol{g})+v_{2} \tau_{22}(\boldsymbol{g})
\end{array}\right]
$$

For the sake of simplicity the numerical scheme is described for the static computational domain $\Omega=\Omega_{0}$. The computational domain $\Omega$ is discretized by the computational mesh $\mathcal{T}_{h}$ consisting of finite number of elements $K \in \mathcal{T}_{h}$. The solution $(\boldsymbol{u}, \boldsymbol{g})$ is sought in $\left[V_{h}^{p}\right]^{4}$ and $\left[V_{h}^{p}\right]^{4 \times 2}$, respectively, where $V_{h}^{p}=\left\{v \in L^{2}(\Omega),\left.v\right|_{K} \in P^{p}(K), \forall K \in \mathcal{T}_{h}\right\}$ with $P^{p}(K)$ being the space of the polynomial functions of degree lower of equal to $p$ on $K, p \geq 1$. The semi-discrete DG formulation is expressed as: find $\boldsymbol{u}_{h} \in\left[V_{h}^{p}\right]^{4}$ and $\boldsymbol{g}_{h} \in\left[V_{p}^{h}\right]^{4 \times 2}$ such that for all $K \in \mathcal{T}_{h}$

$$
\begin{aligned}
& \int_{K} \frac{\partial \boldsymbol{u}_{h}}{\partial t} \cdot \boldsymbol{\varphi} d V+\int_{K}\left(\boldsymbol{F}^{i}\left(\boldsymbol{u}_{h}\right)-\boldsymbol{F}^{v}\left(\boldsymbol{u}_{h}, \boldsymbol{g}_{h}\right)\right): \nabla \boldsymbol{\varphi} d V- \\
&-\int_{\partial K}\left(\boldsymbol{F}^{i *}\left(\boldsymbol{u}_{h}\right)-\boldsymbol{F}^{v *}\left(\boldsymbol{u}_{h}, \boldsymbol{g}_{h}\right)\right) \cdot \boldsymbol{\varphi} d S=\mathbf{0}, \quad \forall \boldsymbol{\varphi} \in\left[P^{p}(K)\right]^{4}, \\
& \int_{K} \boldsymbol{g}_{h}: \boldsymbol{\psi} d V+\int_{K} \boldsymbol{u}_{h} \cdot(\nabla \cdot \boldsymbol{\psi}) d V-\int_{\partial K}\left(\boldsymbol{u}_{h}^{*} \otimes \boldsymbol{n}\right): \boldsymbol{\psi} d S=\mathbf{0}, \quad \forall \boldsymbol{\psi} \in\left[P^{p}(K)\right]^{4 \times 2},
\end{aligned}
$$

where in the surface integrals a numerical fluxes replaced the physical fluxes similarly as in [4]. Both the surface and the volume integrals are approximated using high-order Gaussian quadrature rules, see e.g. [4]. The inviscid fluxes are computed using Roe's method, see [9]. The viscous fluxes, i.e., $\boldsymbol{F}^{\nu}$ in (14) and $\boldsymbol{u}_{h}$ in (15) are discretized with aid of compact discontinuous Galerkin (CDG) method, see [7]. Finally, fluxes in Equations (14) and (15) are modified according to the ALE method, see e.g. [11]. Time integration is done with aid of a high-order Runge-Kutta (RK) method.

\section{Numerical Results}

\subsection{Flow past Cylinder}

To validate the high-order scheme, a test case consisting of the flow-induced vibration of a circular cylinder was considered, where the cylinder is allowed to move in vertical direction, see Figures 1a and 2a. The free stream velocity $\boldsymbol{v}=(1,0) \mathrm{m} / \mathrm{s}$, density $\varrho=1 \mathrm{~kg} / \mathrm{m}^{3}$, Mach number is equal to 0.1 , and a Reynolds number with respect to diameter $D$ is equal to 100 . The constants chosen for this problem were $D=0.1 \mathrm{~m}, m=1 \mathrm{~kg}, k=0.04 \mathrm{~N} / \mathrm{m}, d=10^{-2} k \mathrm{~N} \mathrm{~s} / \mathrm{m}, \nu=0.001 \mathrm{~m}^{2} / \mathrm{s}$ and $l=1 \mathrm{~m}$. 


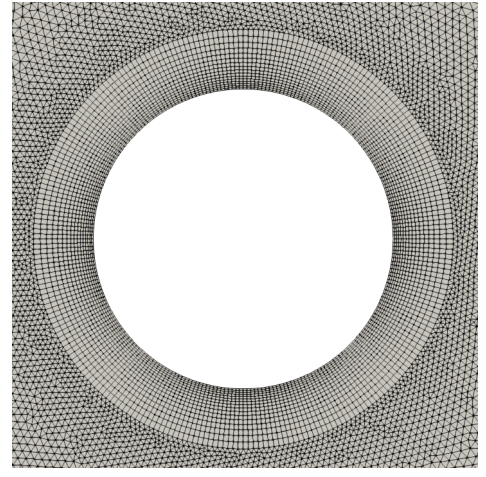

(a) Detail of grid used for FV simulations

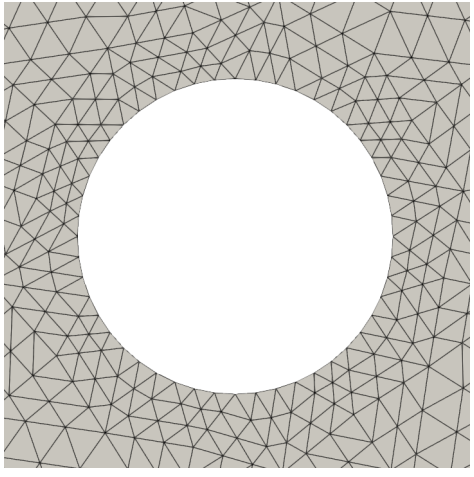

(b) Detail of grid used for DG simulations

Figure 3: Details of the computational meshes for the case of the flow around the cylinder

The FV approximations were obtained on hybrid grid, i.e., quadrilateral cells in vicinity of cylinder and triangles in the rest of the domain with total of 100431 cells, see Figure 3a. The DG approximations were obtained on triangular grid, see Figure 3b, with total of 2051 cells, i.e, 6153 DoF for $p=1$ and 43071 DoF for $p=5$. Figure 4 shows contours of the velocity magnitude at time $t=100 \mathrm{~s}$ of DG approximation obtained with fourth order RK method and fifth order of polynomials $p=5$ (RK4-DG5).

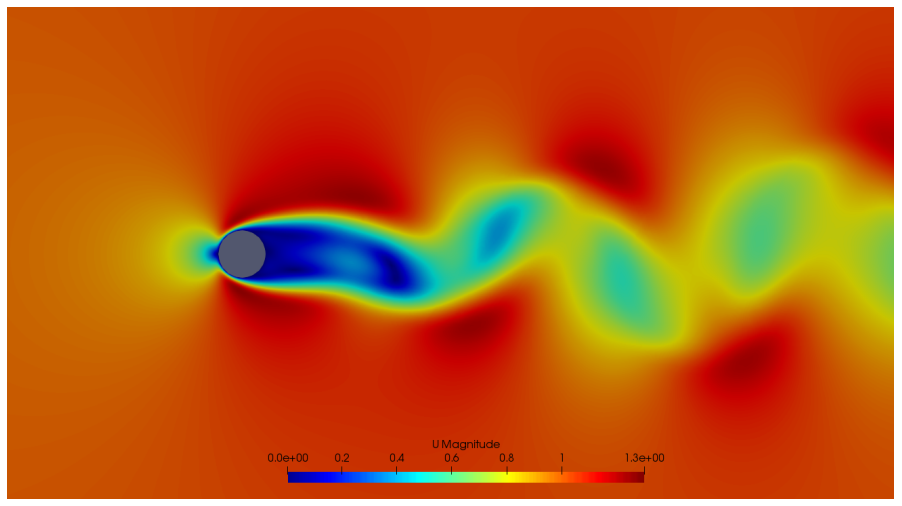

Figure 4: Contours of the velocity magnitude at $t=100 \mathrm{~s}$

Figure 5 shows the dependence of the displacement $h$ on time during the computation. Numerical approximation obtained with RK2-DG1 falls behind both results obtained with RK4-DG5 and FV scheme which shows good match. Taking into context the computational grids (see Figure 3 ) these results shows that FV has much higher grid requirements to obtain acceptable solution as expected.

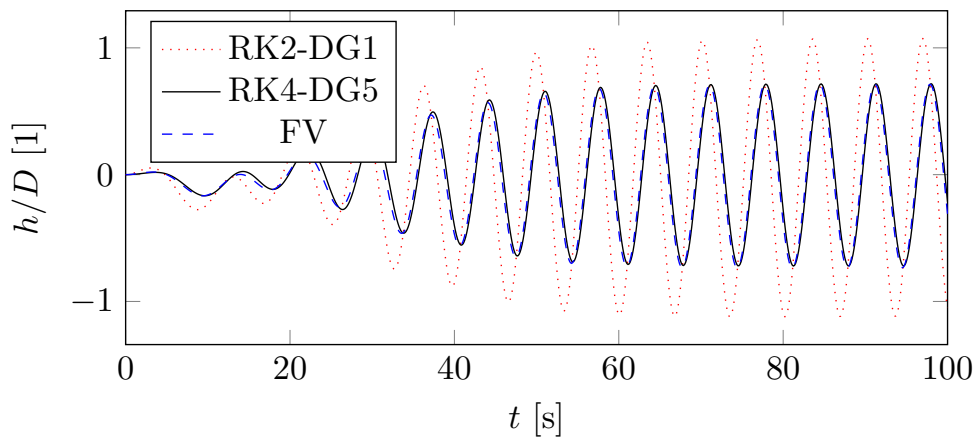

Figure 5: Displacement $h$ during selected time period 


\subsection{Flow past NACA 0012 Airfoil}

This section presents the numerical approximation of the coupled problem of the fluid flow and structure interaction. The described methods were applied to the analysis of flow induced vibrations of the profile NACA 0012. The computation was carried out for the following data: $m=0.086622 \mathrm{~kg}, S_{\alpha}=m e=-0.000779673 \mathrm{~kg} \mathrm{~m}$, where $e=x_{1, \mathrm{~cm}}-x_{1, \text { ea }}$ is the excentricity and $\boldsymbol{x}_{\mathrm{cm}}$ is the center of mass, $I_{\alpha}=0.000487291 \mathrm{~kg} \mathrm{~m}^{2}, k_{h}=105.109 \mathrm{~N} / \mathrm{m}, k_{\alpha}=3.695582 \mathrm{~N} \mathrm{~m} / \mathrm{rad}$, $l=0.05 \mathrm{~m}, c=0.3 \mathrm{~m}, \varrho=1.225 \mathrm{~kg} / \mathrm{m}^{3}, \nu=1.5 \cdot 10^{-5} \mathrm{~m} / \mathrm{s}^{2}$ and the position of the elastic axis $\boldsymbol{x}_{\mathrm{ea}}=(0.4 c, 0)=(0.12,0) \mathrm{m}$. Considered free stream velocity flow velocities are $U_{\infty}=30,35 \mathrm{~m} / \mathrm{s}$, which yield the Reynolds numbers based on chord $R e_{c}=600000,700000$, and Mach numbers $0.21,0.245$. Simulation starts at $t=0$ with the initial condition being the fully developed flow with the airfoil in a fixed position given by the prescribed initial translation $h(0)=-50 \mathrm{~mm}$ and the initial angle of attack $\alpha(0)=6 \mathrm{deg}$. Initial velocities of the structural model are $\dot{h}(0)=0$ and $\dot{\alpha}(0)=0$. The FV approximations were obtained on hybrid grid, i.e., quadrilateral cells in vicinity of airfoil and triangles in the rest of the domain with total of 300952 cells. The DG approximations were obtained on triangular grid with total of 11004 cells, i.e, 231084 DoF for $p=5$.

The results of the airfoil motion due to the fluid-structure interaction for time $t \in\langle 0,1\rangle$ are shown in Figures 6 and 7 for the free stream velocity velocities $U_{\infty}=30,35 \mathrm{~m} / \mathrm{s}$. Approximations obtained with RK4-DG5 and FV scheme shows excellent match.

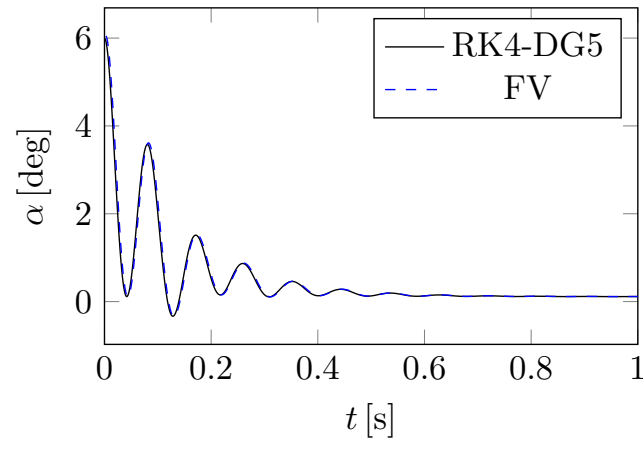

(a) Rotational displacement

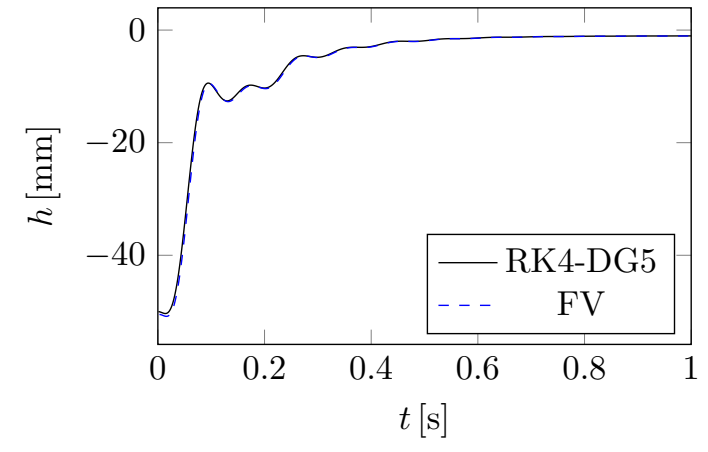

(b) Vertical displacement

Figure 6: Flow induced vibrations for $U_{\infty}=30 \mathrm{~m} / \mathrm{s}$

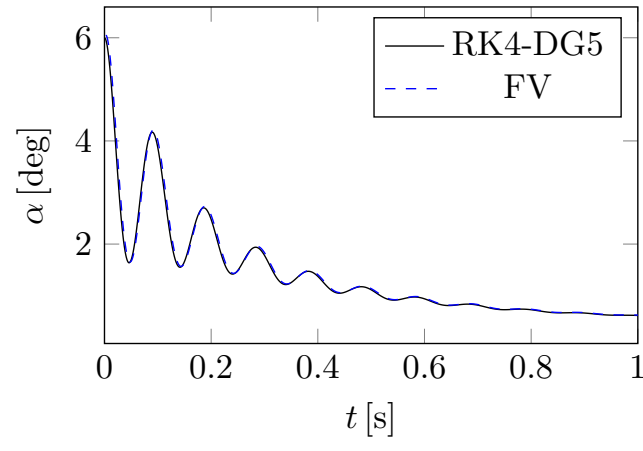

(a) Rotational displacement

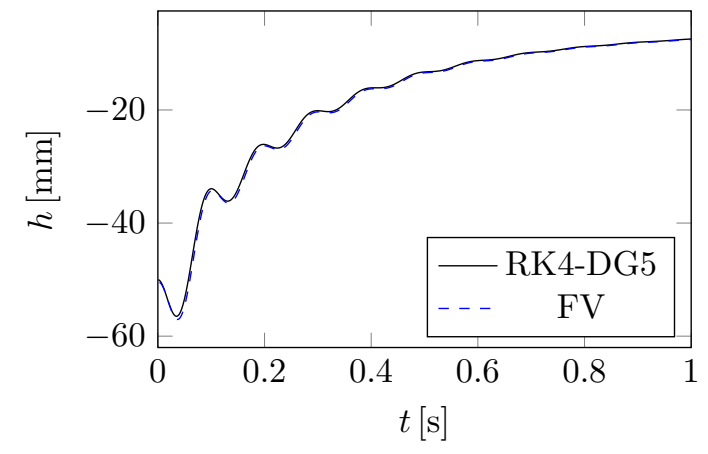

(b) Vertical displacement

Figure 7: Flow induced vibrations for $U_{\infty}=35 \mathrm{~m} / \mathrm{s}$

\section{Conclusion}

In this paper a mathematical model of the compressible fluid flow for the fluid-structure interaction problems is presented and numerically approximated by two numerical methods, i.e., presented high-order discontinuous Galerkin scheme and the second order finite volume scheme. In order to 
address the deformation of the fluid domain the ALE method is used. Both schemes are applied for the solution of two aeroelastic benchmarks problems and the obtained data are compared. All the numerical results indicate that the presented DG scheme is applicable for the solution of an aeroelastic problems.

\section{Acknowledgment}

This work was supported by the Grant Agency of the Czech Technical University in Prague (SGS19/154/ OHK2/3T/12). Authors acknowledge support from the ESIF, EU Operational Programme Research, Development and Education, and from the Center of Advanced Aerospace Technology (CZ.02.1.01/0.0/0.0/ 16_019/0000826), Faculty of Mechanical Engineering, Czech Technical University in Prague.

\section{References}

[1] J. Badur and M. Bryk: Accelerated Start-up of the Steam Turbine by Means of Controlled Cooling Steam Injection. Journal of Energy, 173, 2019.

[2] J. Blažek: Computational Fluid Dynamics: Principles and Applications. Elsevier, third edition, 2015.

[3] J. Fürst: Development of a Coupled Matrix-free LU-SGS Solver for Turbulent Compressible Flows. Computers \& Fluids, 172:332-339, 82018.

[4] Jan S. Hesthaven and Tim Warburton: Nodal Discontinuous Galerkin Methods: Algorithms, Analysis, and Applications. Springer, 2008.

[5] J. Horáček and P. Sváček: Finite Element Simulation of a Gust Response of an Ultralight 2-DOF Airfoil. Proceedings of the ASME 2014 Pressure Vessels \& Piping Conference, 2014.

[6] S.S. Hoseini, G. Najafi, B. Ghobadian, and A.H. Akbarzadeh: Impeller Shape-optimization of Stirred-tank Reactor: CFD and Fluid Structure Interaction Analyses. Chemical Engineering Journal, 413, 2021.

[7] J. Peraire and P.O. Persson: The Compact Discontinuous Galerkin (CDG) Method for Elliptic Problems. SIAM Journal on Scientific Computing, 30(4), 2008.

[8] P. Sváček and J. Horáček: FE Numerical Simulation of Incompressible Airflow in the Glottal Channel Periodically Closed by Self-sustained vocal Folds Vibration. Journal of Computational and Applied Mathematics, 393, 2021.

[9] E.F. Toro: Riemann Solvers and Numerical Methods for Fluid Dynamics. Springer, third edition, 2009.

[10] H. G. Weller, G. Tabor, H. Jasak, and C. Fureby: A Tensorial Approach to Computational Continuum Mechanics Using Object-Oriented Techniques. Computers in Physics, 12(6):620631, 1998.

[11] O. Winter and P. Sváček: On Numerical Simulation of Flexibly Supported Airfoil in Interaction with Incompressible Fluid Flow using Laminar-Turbulence Transition Model. Computers 83 Mathematics with Applications, 83, 2021. 